\title{
RESPUESTA DEL Eucalyptus globulus ssp. globulus A FERTILIZACIONES DE APOYO EN LA VIII REGION
}

\author{
Sergio Calderón Sanchez (*)
}

\section{RESUMEN}

En las zonas húmedas templadas costeras de la provincia de Concepción y húmedas de la Cordillera de los Andes de la provincia de Bio-Bio, Chile, se probaron diversas combinaciones de fertilizantes, aplicadas en dosis subdivididas dentro de los tres primeros años de edad en plantaciones de Eucalyptus globulus ssp. globulus.

Los resultados indican que en el ensayo de la zona costera de la provincia de Concepción las aplicaciones de urea producen la mejor respuesta, tanto en terminos de producción fisica como económica, mostrando una rentabildad superior al $12 \%$.

En el ensayo de la zona precordillerana andina en tanto, la mejor respuesta la ofrece una combinación de $N, P, K$ y $B$, registrando una alta supervivencia y el mayor rendimiento volumétrico. Este tratamiento arroja también una rentabilidad superior al $12 \%$.

Los ensayos confirman que las fertilizaciones de apoyo en serie durante la época juvenil, en plantaciones establecidas en suelos con deficiencias nutricionales, inducen un crecimiento adicional de importancia si la abertura del dosel es suficiente.

Palabras clave: Eucalyptus, Fertilización, Analisis Económico.

\section{ABSTRACT}

Fertilization treatments applied early in the rotation, up to 3 years old, to Eucalyptus globulus ssp. globulus plantations were tested in two climatic zones in Chile: The humid temperate coastal zone of Concepcion and the humid cold Andean area of Bio-Bio province.

Best growth and economic results were obtained with $N$ treatment in the coastal area of Concepcion and with a mixture of $N, P, K$, and $B$ in the andean zone. The cost benefit analysis gives for both trials internal rates of return over $12 \%$.

The results show that early fertilizations, applied in Eucalyptus plantations growing in deficitary soils, can give an important aditional growth and higher rates of survival.

Keywords: Eucalyptus, Fertilization, Cost-Benefut Analysis.

(*) Ingeniero Forestal. División Silvicultura. Instituto Forestal. Huérfanos 554. Casilla 3085. Santiago. 


\section{INTRODUCCION}

La corrección de deficiencias nutricionales en los suelos forestales, generalmente ha sido abordada mediante enmiendas que se efectúan por una sola vez, al momento de la plantación. Sin embargo, numerosos ensayos en otros paises (Hunter, I.R. y otros . 1986), demuestran que las fertilizaciones de apoyo, aplicadas en serie durante la época juvenil de la plantación, pueden inducir un crecimiento adicional de tal magnitud que es posible considerarlas con una herramienta más en el mejoramiento del manejo. La literatura demuestra que la respuesta de Eucalyptus globulus a los tratamientos con fertilizantes es dependiente de la edad del rodal, la carencia de determinado elemento en el suelo y del grado de abertura del dosel provocado por las intervenciones silvícolas de podas o raleo. En suma, habiéndo deficiencias en el suelo será la juventud y el espacio disponible para que se desarrollen las raices y la copa, los que determinarán el grado de respuesta al fertilizante.

El objetivo de este estudio es evaluar la mejor combinación y dosis de fertilizantes de apoyo para el Eucalyptus globulus en la zona costera de la provincia de Concepción y en la zona pre-cordillerana de la provincia de Bio-Bio.

La evaluación presentada en este estudio se realiza a través de un análisis de varianza de los tratamientos aplicados para establecer con certeza estadística, cual es el mejor tratamiento de fertilización eincluye las pruebas de Tukey, método de comparación múltiple que permite individualizar la significación de las diferencias entre tratamientos. Sin embargo, la decisión última del mejor tratamiento a aplicar es una decisión económica, razón por la cual se incluye una proyección del crecimiento hasta la edad de rotación y un análisis del costo e ingreso marginal generado por la aplicación del fertilizante que presenta mejor respuesta volumétrica. Este mismo tratamiento es el que posteriormente resulta ser tambien el mejor desde el punto de vista económico.

\section{METODOLOGIA}

En junio de 1986 se establecieron dos ensayos; uno en la provincia de Concepción y otro en la de Bio-Bio, el primero con un diseño experimental al azar y el otro factorial de 6 y 4 repeticiones, respectivamente, y parceladas de 25 plantas espaciadas a 3 por $3 \mathrm{~m}$. Mayores antecedentes de la instalación se encuentran en la publicación del IV Informe de Actividades del Instituto Forestal (1988). En cada lugar de ensayo se aplicaron 18 combinaciones de fertilizantes, tales como úrea, superfosfato triple y ácido bórico, en la provincia de Concepción, y úrea, superfosfato triple boronatrocalcita y sulfato de potasio en la provincia de Bio-Bio. La periodicidad de las aplicaciones fue similar en los dos lugares. Se aplicó la primera dosis en Septiembre de 1986, la segunda en AgostoSeptiembre de 1987, la tercera en Mayo de 1988 y la cuarta en Agosto de 1988. Los fertilizantes fueron aplicados en forma localizada a $20 \mathrm{~cm}$ de profundidad y en dos zanjas 
ubicadas paralelamente a $26 \mathrm{~cm}$ de la planta.

Los tratamientos en la zona costera de la provincia de Concepción (Apéndice 1) consisten en distintas combinaciones de los siguientes compuestos y dósis por planta.
$\mathrm{N} 1=50$ gr de Urea
$\mathrm{N} 2=100$ gr de Urea
$\mathrm{Pl}=40 \mathrm{gr}$ de Superfosfato triple.
$\mathrm{B} 1=20 \mathrm{gr}$ de Acido bórico.
$\mathrm{B} 2=40 \mathrm{gr}$ de Acido bórico.

Los tratamientos aplicados en los suelos de trumao de la precordillera de Los Andes en la provincia de Bio-Bio, consisten en combinaciones de los siguientes compuestos:

$$
\begin{aligned}
& \mathrm{N} 1=50 \mathrm{gr} \text { de Urea } \\
& \mathrm{N} 2=80 \mathrm{gr} \text { de Urea } \\
& \mathrm{P} 1=40 \mathrm{gr} \text { de Superfosfato triple. } \\
& \mathrm{B} 1=30 \mathrm{gr} \text { de Boronatrocalcita. } \\
& \mathrm{K} 1=50 \mathrm{gr} \text { de Sulfato de potasio. }
\end{aligned}
$$

El efecto de los fertilizantes se refleja tanto en el crecimiento diamétrico a la altura del pecho como en la altura total del árbol y la supervivencia de las plantas. Los controles efectuados al año 3 y en especial al año 5 se consideran valiosos para reflejar el efecto acumulado de las cuatro aplicaciones hechas en los 3 primeros años de la plantación. También se incluye el análisis estadístico de un índice combinado de las variables DAP y altura $\left(D^{2} P^{2} \mathrm{H}\right)$, el cual podría expresar en mejor forma el crecimiento.

El estudio estadístico incluye un análisis de varianza (ANDEVA) que adjudica la proporción de las diferencias observadas que se debe al tratamiento por fertilización, al efecto bloques o al error experimental y los compara con valores tabulados para decidir si estas diferencias son significativas. La prueba adicional de Tukey especifica la diferencia que existe entre cada tratamiento y mide el nivel de significación.

Los datos observados al quinto año son proyectados en los 15 y 20 años con el modelo de crecimiento para las plantaciones de Eucalyptus globulus existente en Tasmania (Goodwin N. y Candy S. G. 1986). Considerando que no ha sido validado para las condiciones chilenas, el modelo puede ser no representativo de las condiciones de sitio probadas. Sin embargo, el ejercicio se incluye con carácter demostrativo y en algunos casos dada la magnitud del efecto fertilizante, los valores economicos alcanzados son insensibles a pequeñas variaciones del modelo. 
El análisis económico se aborda con carácter marginal, capitalizando hasta la edad de rotación sólo los costos imputables a la enmienda con fertilizantes, los cuales se comparan con los ingresos también marginales generados por la venta del volumen adicional producidos por el mejor tratamiento.

Para encontrar el mejor desde el punto de vista económico, no sólo el mejor tratamiento en volumen es analizado, sino que todos los tratamientos lo son, para encontrar si alguno presenta menor costo marginal.

Este enfoque económico introduce una limitación muy fuerte al asumir que el uso de fertilizantes sólo cambia el volumen total y su costo y las demás condiciones permanecen constantes. Esto representa una restricción que se aleja de la realidad, en la medida que el parámetro cambiado interactúa con otros de alta significación económica (tamaño de las trozas, calidad del producto, costo de madereo y transportes, etc.).

\section{RESULTADOS Y DISCUSION DE RESULTADOS}

\section{Respuesta en Diámetro.}

Para apreciar la evolución del DAP se debe observar la historia del ensayo. Al tercer año en la provincia de Concepción, este muestra que los dos tratamientos más efectivos son los mismos que en altura. El primero, $56 \mathrm{~kg}$. de urea por hectárea aplicados 4 veces en los 3 primeros años, hace que el DAP crezca hasta $8,1 \mathrm{~cm}$, en vez de $5,5 \mathrm{~cm}$ obtenido como promedio por las parcelas testigo. Aún cuando esta diferencia no es significativa, presenta un crecimiento en área basal de $2,39 \mathrm{~m}^{2} /$ ha en relación a los rodales sin aplicación, que presentan un área basal de $2,06 \mathrm{~m}^{2} / \mathrm{ha}$. El segundo tratamiento más efectivo es el de $100 \mathrm{gr}$ de urea $+40 \mathrm{gr}$ de ácido bórico por planta el que iguala los crecimientos en DAP obtenidos por el tratamiento de urea sola.

En 1991, al quinto año de la plantación (Cuadro № 1) el primer y segundo tratamiento trastocan su posición resultando que $100 \mathrm{gr}$ de urea $+40 \mathrm{gr}$ de ácido bórico presenta un DAP promedio de $12,9 \mathrm{~cm}$, superando en $3,0 \mathrm{~cm}$ el tratamiento testigo. Esta diferencia aunque no significativa, representa un incremento en área basal de 56,9\% por hectárea. 
Cuadro 1

ZONA COSTERA-SUR DE LA PROVINCIA DE CONCEPCION. EDAD 5 AÑOS

\begin{tabular}{|c|c|c|}
\hline TRATAMIENTO & DIAMETRO $(\mathrm{cm})$ & ALTURA $(\mathrm{m})$ \\
\hline 1 & 10,38 & 10,47 \\
2 & 12,33 & 12,28 \\
3 & 10,06 & 11,17 \\
5 & 12,56 & 14,03 \\
6 & 11,76 & 12,58 \\
7 & 10,04 & 10,29 \\
8 & 9,90 & 11,36 \\
9 & 11,90 & 12,73 \\
10 & 12,93 & 13,23 \\
11 & 11,14 & 11,67 \\
12 & 11,44 & 11,86 \\
13 & 12,55 & 13,04 \\
14 & 10,54 & 12,37 \\
15 & 11,85 & 12,42 \\
16 & 9,70 & 10,98 \\
17 & 9,88 & 11,17 \\
18 & 9,90 & 10,63 \\
& 9,36 & 10,69 \\
\hline
\end{tabular}

El segundo tratamiento resulta ser $56 \mathrm{Kg}$ ha de urea lo que demuestra que los suelos de la cordillera de la costa en la parte sur de la provincia de Concepción son deficitarios principalmente en nitrógeno.

En la zona de suelos de trumao de la precordillera de Los Andes, en la provincia de Bio-Bio, los tratamientos con los mejores crecimientos en DAP a los 5 años (Cuadro 2) son (N1P1B1K1) y (N2P1B1K1), los mismos que presentan el mejor crecimiento en altura, es decir, las dos variables comprueban que estos suelos son deficitarios en nitrógeno, fósforo, boro y potasio. 
Cuadro № 2

ZONA PRECORDILLERANA DE LOS ANDES DE LA PROVINCIA DE BIO-BIO. EDAD 5 AÑOS

\begin{tabular}{|c|c|c|}
\hline TRATAMIENTO & DIAMETRO $(\mathrm{cm})$ & ALTURA (m) \\
\hline 1 & 10,39 & 7,62 \\
\hline 2 & 9,80 & 7,87 \\
\hline 3 & 8,34 & 6,75 \\
\hline 4 & 8,90 & 7,21 \\
\hline 5 & 12,39 & 9,94 \\
\hline 6 & 10,28 & 8,92 \\
\hline 7 & 10,37 & 7,49 \\
\hline 8 & 10,68 & 8,41 \\
\hline 9 & 11,66 & 9,72 \\
\hline 10 & 10,87 & 9,11 \\
\hline 11 & 9,76 & 8,00 \\
\hline 12 & 9,44 & 7,93 \\
\hline 13 & 9,00 & 7,78 \\
\hline 14 & 9,82 & 8,25 \\
\hline 15 & 9,92 & 7,48 \\
\hline 16 & 8,51 & 7,22 \\
\hline 17 & 9,95 & 7,43 \\
\hline 18 & 9,84 & 8,39 \\
\hline
\end{tabular}

\section{Respuesta en altura}

Una de las formas más eficaces de observar el efecto de los fertilizantes en el corto plazo, es medir el crecimiento del follaje, pues en este componente de la biomasa donde primero se refleja el efecto fertilización, especialmente si se trata de compuestos nitrogenados. Por esta razón, la altura del árbol al incluir el incremento del ápice resulta ser el mejor indicador. En el análisis de varianza de altura al tercer año para la zona costerasur de la provincia de Concepción, el tratamiento de $56 \mathrm{Kg}$ de urea aplicado 3 meses después de la plantación, con repetición de la misma dósis a los 14, 23 y 26 meses, es significativamente diferente al testigo (sin aplicación) y entrega la mejor respuesta. El mismo tratamiento es también el mejor al quinto año de observación (Cuadro № 1). La diferencia en altura de este tratamiento (4) es $3.2 \mathrm{~m}$ superior a $10.8 \mathrm{~m}$ promedio del testigo y no es significativa. La evolución de la altura para los 3 mejores tratamientos en la zona costera sur de la provincia de Concepción se presenta en el Gráfico № 1 . 


\section{Grafico $N^{2} 1$ \\ ENSAYO DE FERTILIZACION - PROVINCIA DE CONCEPCION ZONA COSTERA-SUR}
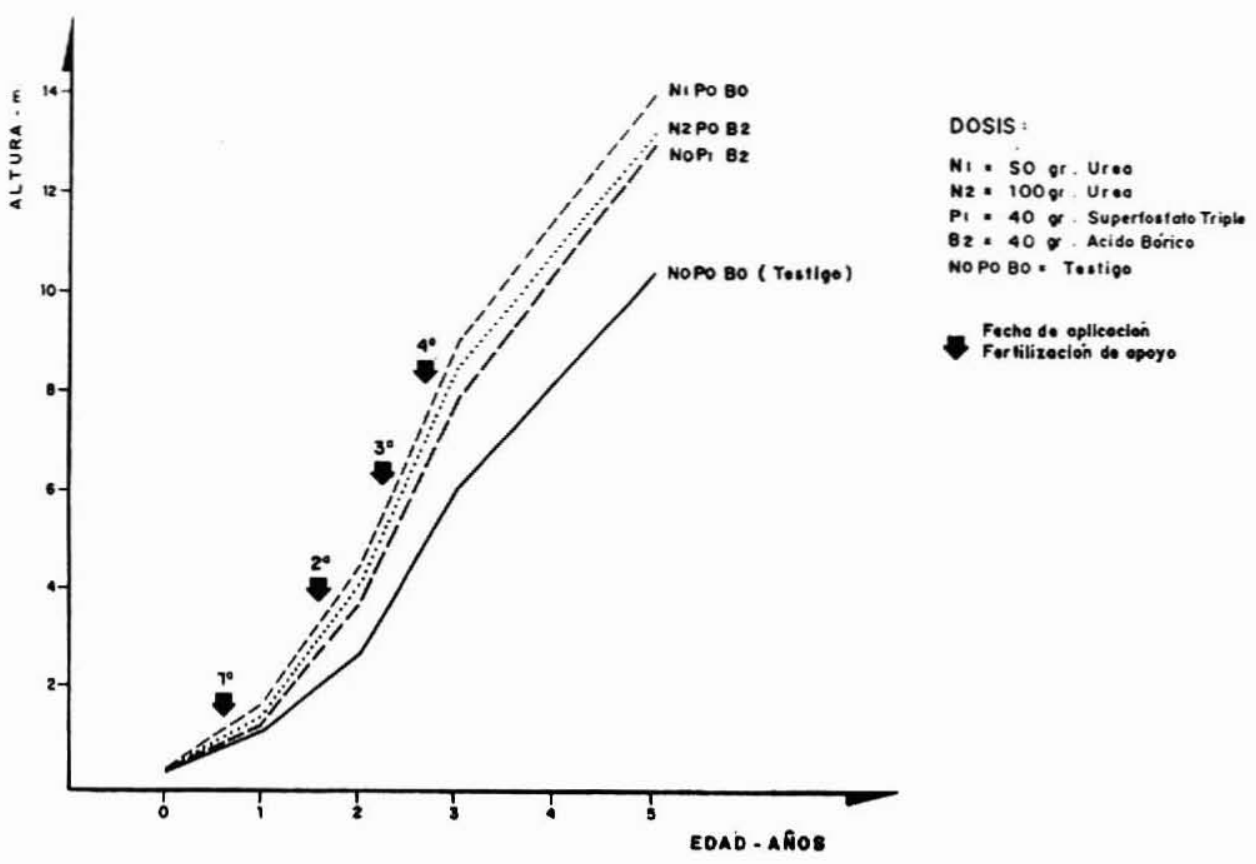

En la zona de la precordillera de Los Andes de la provincia de Bio-Bio a los 5 affos de edad (Cuadro $N^{2}$ 2) el tratamiento (N1P1B1K1) $56 \mathrm{Kg} / \mathrm{ha}$ de urea, 44,5 Kg/ha de superfosfato triple, $33,3 \mathrm{Kg} / \mathrm{ha}$ de boronatrocalcita y $56 \mathrm{Kg} / \mathrm{ha}$ de sulfato de potasio presenta el mejor crecimiento en altura alcanzando $9,9 \mathrm{~m}$ contra 7,4 m del tratamiento testigo. esta diferencia es significativa al aplicar la prueba de Tukey. El segundo mejor tratamiento aunque no significativamente diferente al testigo, es el N2P1B1K1. Estos dos primeros tratamientos son los mismos que resultan de mejor crecimiento en diámetro, lo cual confirma que los suelos de trumao en la precordillera de Los Andes son deficitarios en nitrogeno, fósforo, boro y potasio y pueden ser corregidos por la agregación de estos elementos. La evolución en altura de los tres mejores tratamientos con respecto al promedio del testigo se presenta en el Gráfico № 2 . 


\section{Gráfico № 2}

ENSAYO DE FERTILIZACION - PROVINCIA DE BIO-BIO ZONA TRUMAO CORDILLERA DE LOS ANDES

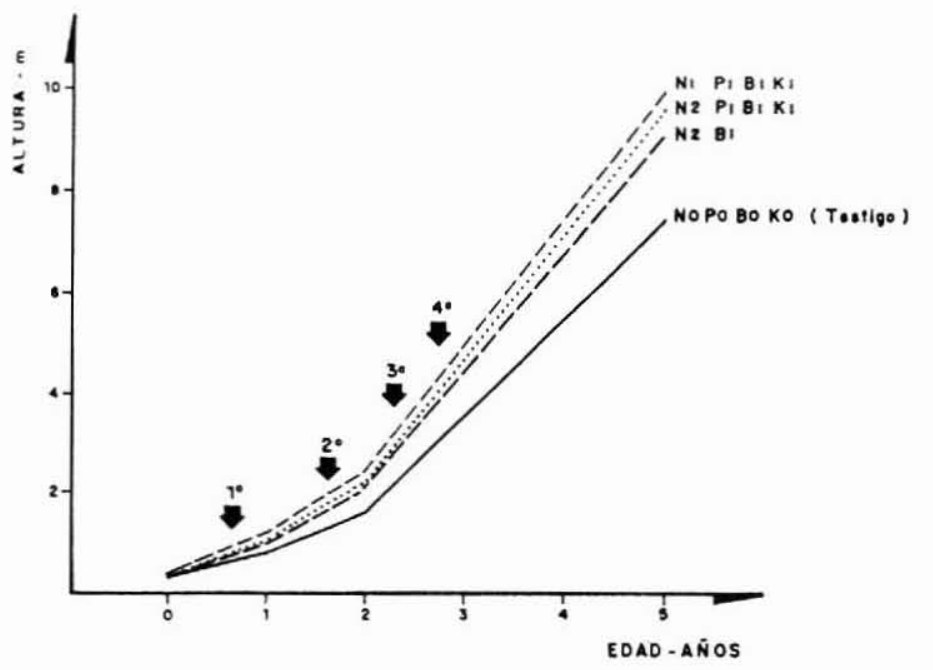

DOSIS

N1 $=50$ or. Se Ureo

N2 = 100 or. de Ureo

$P_{1}=4 \sigma$ or. Superfostoto Triple

$B_{1}$ - $30 \mathrm{gr}$. Botonato Calcito

KI = 50 or. Sulfoto of Potozlo

Fecho de oplicesion

Fortilizoción do opoyo

Como el fertilizante mejora el crecimiento en Diámetro y en Altura, está demás decir que hay diferencias significativas en el factor $D^{2} P^{2} \mathrm{H}$.

Un mejoramiento adicional, muchas veces olvidado, es que las plantas de mejor crecimiento también resultan con una mayor supervivencia. Este efecto, aunque no significativo, se observa en los suelos de trumao.

\section{PROYECCIONES FUTURAS}

Los resultados en la provincia de Concepción, son proyectados en el Cuadro 3 para una rotación de 15 y 20 años usando un modelo de crecimiento de $\boldsymbol{E}$. Globulus en Tasmania. (Goodwin N. y Candy S.G. 1986). 


\section{Cuadro No 3}

PROYECCION DEL CRECIMIENTO CON Y SIN USO DE UREA EN LA ZONA COSTERA-SUR DE LA PROVINCIA DE CONCEPCION

\begin{tabular}{|c|c|c|c|}
\hline \multirow{2}{*}{\multicolumn{2}{|c|}{ VARIABLE }} & \multicolumn{2}{|c|}{ TRATAMIENTO } \\
\hline & & \multirow[t]{2}{*}{ UREA } & \multirow{2}{*}{ TESTIGO } \\
\hline & Аก̃o 3 & & \\
\hline DAP cm & & 8.10 & 5,54 \\
\hline Altura total $\mathrm{m}$ & & 9,02 & 6,18 \\
\hline & Aก̃o 5 & & \\
\hline DAP cm & & 12,56 & 9,84 \\
\hline Altura total m & & 14,03 & 10,79 \\
\hline Vol por árbol m³ ssc (Lisboa) & & 0,03677 & 0,00555 \\
\hline Vol por arbol m³ssc (Diaz) & & 0,05905 & 0,02787 \\
\hline Vol por árbol promedio (Concepción) & ---- & 0.04791 & 0.01671 \\
\hline Vol por árbol m³ ssc (Tasmania) & & 0,03972 & 0,01875 \\
\hline PROYECCION FUTURA & & & \\
\hline DAP cm & 10 años & 18,58 & 14,56 \\
\hline DAP cm & 12 años & 19,92 & 15,23 \\
\hline DAP cm & 15 años & 22,00 & 17,24 \\
\hline DAP $\mathrm{cm}$ & 20 años & 24,50 & 19,20 \\
\hline Altura $\mathrm{m}$ & 10 años & 27,76 & 27,72 \\
\hline Altura $\mathrm{m}$ & 12 años & 31,22 & 31,18 \\
\hline Altura $\mathrm{m}$ & 15 años & 36,04 & 36,00 \\
\hline Altura m & 20 años & 43,38 & 43,33 \\
\hline Vol por árbol $\mathrm{m}^{3} \mathrm{ssc}$ & 12 años & 0,32552 & 0,19004 \\
\hline Vol por árbol m³ ssc & 15 años & 0,48808 & 0,29939 \\
\hline Vol por árbol m³ ssc & 20 años & 0,78080 & 0.47897 \\
\hline Densidad árbol/ha & 5 años & 864 & 856 \\
\hline Densidad árbol/ha & 10 años & 864 & 856 \\
\hline Densidad árbol/ha & 15 años & 799 & 780 \\
\hline Densidad árbol/ha & 20 años & 532 & 658 \\
\hline Vol total $\mathrm{m}^{3}$ ha ssc & 15 años & 390,0 & 233.52 \\
\hline Vol com $\mathrm{m}^{3} /$ ha ssc & 15 años & $374,40(96 \% \mathrm{VT})$ & $205,50(88 \% \mathrm{VT})$ \\
\hline Vol total $\mathrm{m}^{2} /$ ha ssc & 20 años & 415,39 & 315,16 \\
\hline Vol com m³/ha ssc & 20 años & $394,62(95 \% \mathrm{VT})$ & $283,64(90 \% \mathrm{VT})$ \\
\hline
\end{tabular}


Es interesante comparar el volumen por árbol a los 5 años obtenido como promedio, para la zona de Concepción y en el modelo de Tasmania. La similitud de valores indica una relativa seguridad para las proyecciones futuras.

En el cálculo de la mortalidad se utilizó como modelo la ecuación de raleo natural, nominada por su coeficiente de $3 / 2$, curva que representa la mortalidad natural obtenida por competencia en bosques no manejados (Goodwin N. y Candy S.G. 1986).

La proyección de crecimiento para el Eucalyptus globulus plantado en la zona de la precordillera de Los Andes provincia de Bio-Bio, se incluye en el Apéndice 2. Las rotaciones consideradas también son de 15 a 20 años, pero el tratamiento testigo sin fertilización se compara con el tratamiento fertilizado con nitrógeno, fósforo, boro y potasio en las dosis más bajas y efectivas.

\section{ANALISIS ECONOMICO}

Conociendo la respuesta volumétrica producida por el mejor tratamiento con fertilizantes a una edad de rotación, lo que falta por establecer es el valor económico de este mejoramiento. A continuación (Cuadro 4) se incluye el análisis financiero, que evalúa el costo de equilibrio para la madera en pie de rotaciones de 15 y 20 años y que permite cubrir los costos incurridos por la aplicación de las fertilizaciones, cuando estos son capitalizados al 8 y $12 \%$ anual 


\section{Cuadro 4}

\section{ANALISIS FINANCIERO MARGINAL PARA EL TRATAMIENTO DE $56 \mathrm{Kg} / \mathrm{Ha}$} DE UREA APLICADO POR 4 VECES

\begin{tabular}{|c|c|c|c|c|c|c|}
\hline & \multirow{3}{*}{ AÑO APLICACION DE ENMIENDA } & \multirow{3}{*}{$\begin{array}{c}\text { COSTO } \\
\text { ACTUAL } \\
\$ / \mathrm{Ha}\end{array}$} & \multicolumn{4}{|c|}{ COSTO CAPITALIZADO } \\
\hline & & & \multicolumn{2}{|c|}{ AÑO 15} & \multicolumn{2}{|c|}{ AÑO 20} \\
\hline & & & $8 \%$ & $12 \%$ & $8 \%$ & $12 \%$ \\
\hline \multirow[t]{4}{*}{0} & 1,5 jornadas + leyes soc. & 6.000 & & & & \\
\hline & $56 \mathrm{Kg}$ urea $(96 \$ / \mathrm{Kg})$ & 5.376 & & & & \\
\hline & $1 / 20$ jornadas supervision & 300 & & & & \\
\hline & & 11.676 & 37.038 & 63.909 & 54.421 & 112.630 \\
\hline \multirow[t]{4}{*}{1} & 3 jornadas + leyes soc. & 12.000 & & & & \\
\hline & $112 \mathrm{Kg}$ urea $(96 \$ / \mathrm{Kg})$ & 10.752 & & & & \\
\hline & $1 / 20$ jornadas supervision & 600 & & & & \\
\hline & & 23.352 & 68.589 & 114.124 & 100.780 & 201.125 \\
\hline \multirow[t]{5}{*}{2} & 1,5 jornadas + leyes soc. & 6.000 & & & & \\
\hline & $56 \mathrm{Kg}$ urea $(96 \$ / \mathrm{Kg})$ & 5.376 & & & & \\
\hline & 1/20 jornadas supervisión & 300 & & & & \\
\hline & & 11.676 & 31.754 & 50.948 & 46.658 & 89.788 \\
\hline & & GRAN TOTAL & 137.381 & 228.981 & 201.859 & 403.543 \\
\hline \multicolumn{3}{|c|}{ COSTO DE EQUILIBRIO $\$ / m^{3}$ EN PIE (*) } & 813.4 & 1355.7 & 1818.5 & 3635.5 \\
\hline \multicolumn{2}{|c|}{ BENEFICIO NETO = TASA DE INTERES } & + & 65.1 & 162.7 & 145.5 & 436.3 \\
\hline \multicolumn{3}{|c|}{ PRECIO DE EQUILIBRIO \$/m EN PIE } & 678.5 & 1518.4 & 1964.0 & 4071.8 \\
\hline
\end{tabular}

Una forma válida de interpretar el análisis es cuncluir que las tasas de capitalización corresponden a la tasa interna de retorno (TIR) cuando el mercado paga los costos de equilibrio $+8 \%$ de beneficio neto.

Actualmente (Junio 1991) las inversiones a 20 años plazo en fertilizaciones con $56 \mathrm{Kg} / \mathrm{ha}$ de urea en la zona costera-sur provincia de Concepción rinden una tasa interna de retorno de $12 \%$ si se considera $\$ 4071,8 / \mathrm{m}^{3}$ (US\$ $12,00 / \mathrm{m}^{3}$ ) como valor de mercado de la madera en pie.

Este análisis económico evalúa desde el punto de vista financiero el tratamiento de urea ( el mejor en volumen). Lo importante es establecer con toda seguridad si este tratamiento representa el mejor desde el punto de vista económico. Para ello, es necesario 
revisar el costo de los demás tratamientos que, aunque de menor respuesta volumétrica, podrían presentar una mayor eficiencia económica. Este análisis se facilita por tener el boro un mayor precio que el nitrógeno y por tener un menor rendimiento volumétrico. Esto descalifica 9 tratamientos que incluyen boro. El tratamiento de doble dósis de nitrógeno (N2 PO BO) resulta también más costoso y no sobrepasa el rendimiento del nitrógeno simple (NI PO BO). El único tratamiento que aparece competitivo es el de 40 gr de superfosfato triple (NO PI BO) por ser producto levemente más barato, lo cual podría compensar el menor rendimiento volumétrico. Sin embargo, efectuadas las proyecciones volumétricas y el cálculo de los costos de equilibrio (Apéndice 3), el tratamiento con urea sigue siendo el mejor.

El análisis financiero para el mejor tratamiento de fertilización ensayado en los suelos de trumao en la cordillera de Los Andes provincia de Bio-Bio, es incluido en el Apéndice 4. Se observa que las ganancias volumétricas son $244 \mathrm{~m}^{3}$ a los 15 años y 317 $\mathrm{m}^{3}$ a los 20 . Estos volúmenes son más altos en la provincia de Concepción pero el costo del tratamiento es más del doble, pues incluye la enmienda de 4 nutrientes principales; nitrógeno, fósforo, boro y potasio.

El precio de equilibrio resultante de $\$ 3.105 / \mathrm{m}^{3}$ con la tasa de interés de $12 \%$, es más bajo que el de Concepción, e indica que aún si el mercado paga US $\$ 9.00 / \mathrm{m}^{3}$ las fertilizaciones en la provincia de Bio-Bio en suelos de trumao, son una alternativa viable.

\section{CONCLUSIONES Y RECOMENDACIONES}

Al comparar las zonas de ensayo de la provincia Concepción y de la provincia de Bio-Bio, las diferencias de sitio previstas al programar los tratamientos se ven confirmadas por los resultados. Es interesante observar que en la localidad de la provincia de Concepción el testigo posee un índice de sitio de $10.8 \mathrm{~m}$ de altura a los 5 años contra 7.6 $\mathrm{m}$ en la provincia de Bio-Bio a la misma edad, indicando un nivel de fertilidad mayor. Sin embargo, este aumento de fertilidad no se traduce en igual ganancia volumétrica por superficie, pues la supervivencia disminuye (probablemente por mayor competencia con malezas por aumento de fertilidad significando en total una pérdida del mayor potencial productivo.

En la zona costera-sur de la provincia de Concepción la respuesta con urea aplicada a plantaciones de Eucalyptus globulus durante los 3 primeros años de la rotación resulta economicamente favorable, con una tasa interna de retorno muy superior al $12 \%$ cuando se proyectan las ganancias (con análisis marginal) hasta edades de corta de 15 años. Esperar hasta los 20 años para realizar los beneficios significa obtener con un precio equivalente a US $\$ 12 / \mathrm{m}^{3}$ de la madera en pie, una tasa interna del retorno de las inversiones en fertilización del $12 \%$. Al comparar la economía de las fertilizaciones con urea con los demás tratamientos, en especial la enmienda simple con superfosfato triple, aún sigue 
siendo el mejor el tratamiento con urea. La conclusión para esta zona es la de preferir la rotación de 15 años cuando las fertilizaciones de urea producen un beneficio mayor.

Esto no debe considerarse definitivo hasta no validar, con un modelo de crecimiento representativo, las proyecciones.

Se recomienda que futuros ensayos de esta naturaleza incluyan tratamientos diferenciados en alguna progresión matemática, de tal modo que se pueda ajustar funciones para calcular el optimo. Por otra parte, la validez de las conclusiones será aumentada cuando el enfoque economico deje de ser marginal y valore todas las mejoras y costos adicionales que surgen al cambiar el diámetro y altura (volumen y tamaño del producto, calidad del producto, reducción de costos de aprovechamiento y transporte, coeficientes de conversión, etc.).

Las enmiendas con nitrógeno, fósforo, boro y potasio en suelos de trumao Cordillera de Los Andes son las mejores y significativamente demuestran un déficit de estos nutrientes en la provincia de Bio-Bio. La factibilidad económica de la aplicación es obvia, dado que el ingreso adicional producido es mayor que en Concepción (producto del mejoramiento obtenido en la supervivencia) y el costo marginal total, aunque evidentemente superior, no es suficientemente alto a los 20 años como para significar un mayor precio. Por esta razon se concluye que la tasa interna de retorno (TIR) para inversiones a 20 años es $12 \%$ cuando el precio del mercado alcanza a $\$ 3.105 / \mathrm{m}^{3}$ (US\$ $9.00 / \mathrm{m}^{3}$ ) para el valor de la madera en pie.

\section{REFERENCIAS BIBLIOGRAFICAS}

Goodwin N. y Candy S.G. 1986. Single-tree and Stand Growth Models for a plantation of Eucalyptus globulus Labill. in Northern Tasmania. Aust. For. Res., 16. 131-44.

Gordon, A.; Graham, J.D. 1986. Changes in Pinus radiata stem form in response to nitrogen and phosphorus fertiliser. New Zealand Journal of Forestry Science. Vol. 16.

Hunter, I.R.; Graham, J.D.; Prince, J.M.; Nicholson, G.M. 1986. Whats site factors determine the 4 year basal area response of Pinus radiata to nitrogen fertiliser. New Zealand Journal of Forestry Science. Vol. 16.

INFOR 1988. IV Informe de actividades Período Abril 1987-Junio 1988 Proyecto Manejo Silvicola del Género Eucalyptus. CORFO. junio.

Prado, J.A. y Barros, S. 1989. Eucalyptus. Principios de Silvicultura y manejo INFOR. CORFO Santiago, Chile. 
Apéndice 1

LISTA DE TRATAMIENTOS

ENSAYADOS EN LA

ZONA COSTERA-SUR DE LA

PROVINCIA DE CONCEPCION
LISTA DE TRATAMIENTOS

ENSAYADOS EN LA ZONA

CORDILLERANA DE LOS ANDES

DE LA PROVINCIA DE BIO-BIO

\begin{tabular}{|c|c|c|c|}
\hline FILA N ${ }^{2}$ & \multicolumn{3}{|c|}{ TRATAMIENTO } \\
\hline 1 & \multicolumn{3}{|c|}{ TESTIGO } \\
\hline 2 & NO & $\mathrm{PO}$ & B1 \\
\hline 3 & NO & $\mathrm{PO}$ & B2 \\
\hline 4 & N1 & $\mathrm{PO}$ & BO \\
\hline 5 & N1 & $\mathrm{PO}$ & B1 \\
\hline 6 & N1 & $\mathrm{PO}$ & B2 \\
\hline 7 & N2 & $\mathrm{PO}$ & $\mathrm{BO}$ \\
\hline 8 & N2 & $\mathrm{PO}$ & B1 \\
\hline 9 & N2 & $\mathrm{PO}$ & B2 \\
\hline 10 & NO & $\mathrm{P} 1$ & $\mathrm{BO}$ \\
\hline 11 & NO & P1 & B1 \\
\hline 12 & NO & P1 & B2 \\
\hline 13 & N1 & P1 & $\mathrm{BO}$ \\
\hline 14 & N1 & P1 & B1 \\
\hline 15 & \multicolumn{3}{|c|}{ TESTIGO } \\
\hline 16 & \multicolumn{3}{|c|}{ TESTIGO } \\
\hline 17 & \multicolumn{3}{|c|}{ TESTIGO } \\
\hline 18 & \multicolumn{3}{|c|}{ TESTIGO } \\
\hline
\end{tabular}

\begin{tabular}{|c|cccc|}
\hline FILA N2 $^{2}$ & \multicolumn{4}{|c|}{ TRATAMIENTO } \\
\hline 1 & \multicolumn{4}{|c|}{ TESTIGO } \\
2 & $\mathrm{~N} 1$ & $\mathrm{PO}$ & $\mathrm{BO}$ & $\mathrm{KO}$ \\
3 & $\mathrm{~N} 2$ & $\mathrm{PO}$ & $\mathrm{BO}$ & $\mathrm{KO}$ \\
4 & $\mathrm{~N} 1$ & $\mathrm{P} 1$ & $\mathrm{BO}$ & $\mathrm{KO}$ \\
5 & $\mathrm{~N} 1$ & $\mathrm{P} 1$ & $\mathrm{~B} 1$ & $\mathrm{~K} 1$ \\
6 & $\mathrm{~N} 1$ & $\mathrm{PO}$ & $\mathrm{B} 1$ & $\mathrm{KO}$ \\
7 & $\mathrm{~N} 1$ & $\mathrm{PO}$ & $\mathrm{BO}$ & $\mathrm{K} 1$ \\
8 & $\mathrm{~N} 2$ & $\mathrm{P} 1$ & $\mathrm{BO}$ & $\mathrm{KO}$ \\
9 & $\mathrm{~N} 2$ & $\mathrm{P} 1$ & $\mathrm{~B} 1$ & $\mathrm{~K} 1$ \\
10 & $\mathrm{~N} 2$ & $\mathrm{PO}$ & $\mathrm{B} 1$ & $\mathrm{KO}$ \\
11 & N2 & $\mathrm{PO}$ & $\mathrm{BO}$ & $\mathrm{K} 1$ \\
12 & NO & $\mathrm{P} 1$ & $\mathrm{~B} 1$ & $\mathrm{~K} 1$ \\
13 & NO & $\mathrm{PO}$ & $\mathrm{B} 1$ & $\mathrm{~K} 1$ \\
14 & NO & $\mathrm{P} 1$ & $\mathrm{~B} 1$ & $\mathrm{KO}$ \\
15 & & TESTIGO & \\
16 & & TESTIGO & \\
17 & & TESTIGO & \\
18 & & TESTIGO & \\
\hline
\end{tabular}




\section{Apéndice 2}

PROYECCION DEL CRECIMIENTO CON Y SIN USO DE N1 P1 B1 K1 EN LA ZONA PRECORDILLERANA DE LOS ANDES. PROVINCIA DE BIO-BIO

\begin{tabular}{|c|c|c|}
\hline \multirow{2}{*}{ VARIABLE } & \multicolumn{2}{|c|}{ TRATAMIENTO } \\
\hline & N1 P1 B1 K1 & TESTIGO \\
\hline \multicolumn{3}{|c|}{ AÑO 5} \\
\hline $\begin{array}{l}\text { D.A.P. } \mathrm{cm} \\
\text { Altura total } \mathrm{m} \\
\text { Vol por árbol } \mathrm{m}^{3} \mathrm{ssc} \text {. (Lisboa) } \\
\text { Vol por árbol } \mathrm{m}^{3} \mathrm{ssc} \text {. (Diaz) }\end{array}$ & $\begin{array}{l}12,39 \\
9,94 \\
0,01841 \\
0,04071\end{array}$ & $\begin{array}{l}9,52 \\
7,63 \\
0,00555 \\
0,01241\end{array}$ \\
\hline $\begin{array}{l}\text { Vol por árbol promedio (Bio - Bio) } \\
\text { Vol por árbol m³ ssc. (Tasmania) }\end{array}$ & $\begin{array}{l}0,02955 \\
0,02738\end{array}$ & $\begin{array}{l}0,01200 \\
0,01241\end{array}$ \\
\hline \multicolumn{3}{|c|}{ PROYECCION FUTURA } \\
\hline $\begin{array}{l}\text { D.A.P. cm } 10 \text { años } \\
\text { D.A.P. } \mathrm{cm} 12 \text { años } \\
\text { D.A.P. } \mathrm{cm} 15 \text { años } \\
\text { D.A.P. cm } 20 \text { años }\end{array}$ & $\begin{array}{l}18,33 \\
19,70 \\
21,70 \\
24,18\end{array}$ & $\begin{array}{r}14,08 \\
15,28 \\
16,68 \\
18,57\end{array}$ \\
\hline $\begin{array}{l}\text { Altura total } 10 \text { años } \\
\text { Altura total } 12 \text { años } \\
\text { Altura total } 15 \text { años } \\
\text { Altura total } 20 \text { años }\end{array}$ & $\begin{array}{l}27,75 \\
31,21 \\
36,04 \\
43,37\end{array}$ & $\begin{array}{l}27,72 \\
31,18 \\
35,80 \\
43,32\end{array}$ \\
\hline $\begin{array}{l}\text { Vol por árbol m } \mathrm{m}^{3} \text { ssc. } 12 \text { anos } \\
\text { Vol por árbol m ssc. } 15 \text { años } \\
\text { Vol por árbol } \mathrm{m}^{3} \mathrm{ssc} .20 \text { aగios }\end{array}$ & $\begin{array}{l}0,31820 \\
0,47486 \\
0,76036\end{array}$ & $\begin{array}{l}0,19129 \\
0,27870 \\
0,44795\end{array}$ \\
\hline $\begin{array}{l}\text { Densidad arbol/ha } 5 \text { años } \\
\text { Densidad árbol/ha } 10 \text { años } \\
\text { Densidad árbol/ha } 15 \text { años } \\
\text { Densidad árbol/ha } 20 \text { años }\end{array}$ & $\begin{array}{l}957 \\
957 \\
886 \\
752\end{array}$ & $\begin{array}{l}716 \\
716 \\
668 \\
567\end{array}$ \\
\hline $\begin{array}{l}\text { Vol total } m^{3} \mathrm{ssc} .15 \text { años } \\
\text { Vol com } \mathrm{m}^{3} \mathrm{ssc} .15 \text { años } \\
\text { Vol total } \mathrm{m}^{3} \mathrm{ssc} 20 \text { aก๊os } \\
\text { Vol com } \mathrm{m}^{3} \mathrm{ssc} .20 \text { años }\end{array}$ & $\begin{array}{ll}421 & \\
404 & (96 \%) \\
572 & \\
543 & (95 \%)\end{array}$ & $\begin{array}{ll}186 & \\
160 & (86 \%) \\
254 & \\
226 & (89 \%)\end{array}$ \\
\hline
\end{tabular}


Apéndice 3

ANALISIS FINANCIERO MARGINAL PARA EL TRATAMIENTO DE 44,4 KG/HA DE SUPERFOSFATO TRIPLE APLICADO EN LA PROVINCIA DE CONCEPCION

\begin{tabular}{|c|c|c|c|c|c|c|}
\hline & \multirow{3}{*}{ AÑO APLICACION DE ENMIENDA } & \multirow{3}{*}{$\begin{array}{c}\text { COSTO } \\
\text { ACTUAL } \\
\$ / \mathrm{Ha}\end{array}$} & \multicolumn{4}{|c|}{ COSTO CAPITALIZADO } \\
\hline & & & \multicolumn{2}{|c|}{ AÑO 15} & \multicolumn{2}{|c|}{ AÑO 20} \\
\hline & & & $8 \%$ & $12 \%$ & $8 \%$ & $12 \%$ \\
\hline \multirow[t]{5}{*}{0} & 1 jornada + leyes soc. & 4.000 & & & & \\
\hline & $44 \mathrm{Kg} /$ ha superfosfato $(\$ 85)$ & 3.778 & & & & \\
\hline & $18 \%$ IVA fertilizante & 680 & & & & \\
\hline & 1/20 jornadas supervision & 200 & & & & \\
\hline & & 8.658 & 27.465 & 47.390 & 40.355 & 83.518 \\
\hline \multirow[t]{5}{*}{1} & 1 jornada + leyes soc. & 4.000 & & & & \\
\hline & $44 \mathrm{Kg} / \mathrm{ha}$ superfosfato $(\$ 85)$ & 3.778 & & & & \\
\hline & $18 \%$ IVA fertilizante & 680 & & & & \\
\hline & 1/10 jornadas supervision & 400 & & & & \\
\hline & & 8.858 & 26.018 & 43.290 & 38.228 & 76.292 \\
\hline \multirow[t]{6}{*}{2} & 2 jornadas + leyes soc. & 8.000 & & & & \\
\hline & $88 \mathrm{Kg} / \mathrm{ha}$ superfosfato $(\$ 85)$ & 7.555 & & & & \\
\hline & $18 \%$ IVA fertilizante & 1.360 & & & & \\
\hline & $1 / 10$ jornadas supervision & 800 & & & & \\
\hline & & 17.715 & 48.178 & 77.299 & 70.789 & 136.228 \\
\hline & & GRAN TOTAL & 101.661 & 167.979 & 149.372 & 296.038 \\
\hline \multicolumn{3}{|c|}{ COSTO DE EQUILIBRIO \$/m³ EN PIE } & 3.366 & 5.562 & 3.403 & 6.744 \\
\hline \multicolumn{2}{|c|}{ BENEFICIO NETO = TASA DE INTERES } & + & 269 & 667 & 272 & 809 \\
\hline \multicolumn{2}{|c|}{ PRECIO DE EQUILIBRIO \$/m² EN PIE } & & 3.635 & 6.229 & 3.675 & 7.553 \\
\hline
\end{tabular}


Apéndice 4

ANALISIS FINANCIERO MARGINAL PARA EL

TRATAMIENTO NI PI BI KI APLICADO POR 4 VECES

ZONA DE PRECORDILLERA DE LOS ANDES SUELOS DE TRUMAO

EN LA PROVINCIA DE BIO - BIO

\begin{tabular}{|c|c|c|c|c|c|c|}
\hline & \multirow{3}{*}{ AÑO APLICACION DE ENMIENDA } & \multirow{3}{*}{$\begin{array}{c}\text { COSTO } \\
\text { ACTUAL } \\
\$ / \mathrm{Ha}\end{array}$} & \multicolumn{4}{|c|}{ COSTO CAPITALIZADO } \\
\hline & & & \multicolumn{2}{|c|}{ AÑO 15} & \multicolumn{2}{|c|}{ AÑO 20} \\
\hline & & & $8 \%$ & $12 \%$ & $8 \%$ & $12 \%$ \\
\hline \multirow[t]{7}{*}{0} & 2 jornadas + leyes soc. & 8.000 & & & & \\
\hline & $56 \mathrm{Kg} / \mathrm{ha}$ urea $(\$ 96)$ & 5.376 & & & & \\
\hline & $44,5 \mathrm{~kg} / \mathrm{ha}$ superfosfato triple & 3.783 & & & & \\
\hline & $33,3 \mathrm{~kg} /$ ha boronatrocalcita & 1.931 & & & & \\
\hline & $56 \mathrm{~kg} /$ ha sulfato potasio & 5.936 & & & & \\
\hline & $1 / 20$ jornadas supervision & 400 & & & & \\
\hline & & 25.426 & 80.656 & 139.171 & 118.509 & 245.267 \\
\hline \multirow[t]{7}{*}{1} & 4 jornadas + leyes soc. & 16.000 & & & & \\
\hline & $112 \mathrm{Kg} / \mathrm{ha}$ urea $(\$ 96)$ & 10.752 & & & & \\
\hline & $89 \mathrm{~kg} /$ ha superfosfato triple & 7.566 & & & & \\
\hline & $66,6 \mathrm{~kg} / \mathrm{ha}$ boronatrocalcita & 3.862 & & & & \\
\hline & $112 \mathrm{~kg} /$ ha sulfato potasio & 11.872 & & & & \\
\hline & $1 / 20$ jornadas supervision & 800 & & & & \\
\hline & & 50.852 & 149.362 & 248.519 & 219.462 & 437.976 \\
\hline \multirow[t]{8}{*}{2} & 2 jornadas + leyes soc. & 8.000 & & & & \\
\hline & $56 \mathrm{Kg} / \mathrm{ha}$ urea $(\$ 96)$ & 5.376 & & & & \\
\hline & $44,5 \mathrm{~kg} / \mathrm{ha}$ superfosfato triple & 3.783 & & & & \\
\hline & $33,3 \mathrm{~kg} / \mathrm{ha}$ boronatrocalcita & 1.931 & & & & \\
\hline & $56 \mathrm{~kg} / \mathrm{ha}$ sulfato potasio & 5.936 & & & & \\
\hline & $1 / 20$ jornadas supervision & 400 & & & & \\
\hline & & 25.426 & 69.149 & 110.946 & 101.603 & 195.525 \\
\hline & & GRAN TOTAL & 299.167 & 498.636 & 439.574 & 878.768 \\
\hline \multicolumn{3}{|c|}{ COSTO DE EQUILIBRIO $\$ / m^{3}$ EN PIE } & 1.226 & 2.044 & 1.387 & 2.772 \\
\hline \multicolumn{2}{|c|}{ BENEFICIO NETO = TASA DE INTERES } & + & 98 & 245 & 111 & 333 \\
\hline \multicolumn{2}{|c|}{ PRECIO DE EQUILIBRIO $\$ / m^{\circ}$ EN PIE } & & 1.324 & 2.289 & 1.498 & 3.105 \\
\hline
\end{tabular}

